\title{
A functional polymorphism rs11614913 in microRNA-196a2 is associated with an increased risk of colorectal cancer although not with tumor stage and grade
}

\author{
NA WANG ${ }^{1}$, YAN LI ${ }^{1}$, LING-JUN ZHU ${ }^{2}$, RONG-MIAO ZHOU ${ }^{1}$, WEI JIN ${ }^{3}$, \\ XIAO-QING GUO ${ }^{4}$, CHUN-MEI WANG $^{5}$, ZHI-FENG CHEN $^{6}$ and WEI LIU ${ }^{7}$ \\ ${ }^{1}$ Department of Molecular Biology, The Fourth Hospital of Hebei Medical University, Shijiazhuang, Hebei 050011; \\ ${ }^{2}$ Department of Oncology, The First Affiliated Hospital of Nanjing Medical University, Nanjing, Jiangsu 210029; \\ ${ }^{3}$ Department of Neurology, Hebei Provincial People's Hospital, Shijiazhuang, Hebei 050051, P.R. China; \\ ${ }^{4}$ Division of Genetic and Molecular Toxicology, National Center for Toxicological Research, Jefferson, AR 72079, USA; \\ ${ }^{5}$ School of Life Sciences, The Chinese University of Hong Kong, Shatin, \\ NT, Hong Kong SAR; Departments of ${ }^{6}$ Cancer Prevention and Control, and ${ }^{7}$ Medical Oncology, \\ The Fourth Hospital of Hebei Medical University, Shijiazhuang, Hebei 050011, P.R. China
}

Received January 17, 2013; Accepted July 08, 2013

DOI: $10.3892 / b r .2013 .146$

\begin{abstract}
A C/T polymorphism (rs11614913) was identified in the microRNA (miRNA) 196a2 (miR-196a2) gene and was implicated in the susceptibility to cancer. Numerous studies have investigated its association with the risk of colorectal cancer (CRC). However, the results were inconsistent and inconclusive. The present meta-analysis was conducted based on the results of six published case-control studies comprising 1,754 cases and 2,430 controls (up to November, 2012). Odds ratios (ORs) and $95 \%$ confidence intervals (CIs) were calculated for the allelic and genotypic comparisons following the co-dominant, dominant and recessive genetic models. The Chi-square-based Q-test was used to assess heterogeneity. Egger's test and inverted funnel plots were used to investigate publication bias. Subgroup analysis was also performed. The results demonstrated that almost all the genetic models (except the model of CT vs. TT) indicated a significant association between rs11614913 polymorphism and CRC risk. The subgroup analysis in an Asian population also demonstrated similar results. However, there was no significant association of miR-196a2 rs11614913 polymorphism with the clinical characteristics of CRC patients. Our results confirmed the association of the polymorphism rs11614913 with the risk of CRC, but not with tumor stage and grade.
\end{abstract}

Correspondence to: Professor Wei Liu, Department of Medical Oncology, The Fourth Hospital of Hebei Medical University, 12 Jiankang Road, Shijiazhuang, Hebei 050011, P.R. China E-mail: hebeiliuwei@yahoo.com.cn; hbykdxwn@yahoo.com.cn

Key words: microRNA-196a2, rs11614913, meta-analysis, polymorphism, colorectal cancer

\section{Introduction}

The incidence of colorectal cancer (CRC) has been on the increase and is currently a major cause of cancer-related morbidity and mortality worldwide, with high rates in westernized societies and an increasing rate in developing countries (1). Although studies reported that lifestyle, dietary habits and environmental factors may be involved in the occurrence of CRC $(2,3)$, inheritance was recently recognized as an important factor (4). Approximately one-third of cases appeared to have inheritance as part of their pathogenesis (5). A large twin study demonstrated that inherited factors account for 35\% of CRC cases, whereas shared environmental factors account for $5 \%$ and non-shared environmental factors for the remaining $60 \%$ (6).

MicroRNAs (miRNAs) are an abundant class of small non-coding, single-stranded RNAs of 21-24 nucleotides that form base pairs with target mRNAs and regulate their post-transcriptional functions as tumor suppressors and oncogenes (7-9). Several human studies provided evidence that the presence of single-nucleotide polymorphisms (SNPs) in miRNAs may alter miRNA processing, expression, and/or binding to target mRNA and are another type of genetic variability that may contribute to the susceptibility to cancer development (10-12).

A C/T polymorphism (rs11614913) was located in the stem region opposite the mature miR-196a2 sequence. Previous studies demonstrated that high expression levels of miR-196a2 may promote the migration and invasion of CRC cells and the C allele of rs11614913 polymorphism may affect miR-196a2 expression levels in cancer (13-16). Several previous studies reported the association between rs11614913 polymorphisms and the susceptibility to CRC $(15,17-21)$. However, those studies produced controversial and inconclusive results. Since the statistical power of an individual study may be insufficient 
for the assessment of rs11614913 polymorphisms, integration of data sets may provide improved statistical power and detect significance.

The present meta-analysis was conducted with the aim of addressing inconsistencies in the findings of previous studies. The meta-analysis was based on published case-control studies, in order to assess the association between the miR-196a2 rs11614913 polymorphism and the susceptibility to CRC.

\section{Materials and methods}

Literature search strategy. A search was conducted on PubMed, EMBASE, ScienceDirect, the Foreign Medical Journal Service (FMJS) and the Chinese National Knowledge Infrastructure (CNKI) databases for all genetic association studies on the rs11614913 polymorphism of miR-196a2 and the susceptibility to CRC, published prior to November, 2012. The search used the following keywords and subject terms: miR-196a 2 or microRNA-196a2 or miRNA-196a2, rs11614913 or polymorphism or SNPs and colorectal cancer/carcinoma/neoplasm. The search was limited to English and Chinese language articles. The reference lists were manually examined to further identify potentially relevant studies. The corresponding authors of conference abstracts without sufficient data were contacted via e-mail for additional information.

Selection criteria. Any human-associated study, regardless of sample size, was included if the following criteria were met: i) use of an unrelated case-control design; ii) investigation of the association between rs11614913 polymorphisms of miR-196a2 and the risk of CRC; iii) genotype distribution of the control population in Hardy-Weinberg equilibrium (HWE) [goodness-of-fit test, degree of freedom $(\mathrm{df})=1$ ] and iv) published in English or Chinese. For articles with the same population resource or overlapping data sets, the publication reporting the largest or most recent data set was included. Therefore, the data for this meta-analysis were obtained from six case-control studies, including a total of 1,754 CRC cases and 2,430 controls.

Data extraction. Two investigators (Xiao-qing Guo and Chun-mei Wang) independently extracted data and reached a consensus on all the items. The following information was recorded for each study: first author, year of publication, country of origin, cancer type, ethnicity, number of cases and controls, study design, genotyping methods and evidence of HWE. For subjects of different ethnicities, data were extracted separately and classified as European or Asian (Table I).

Statistical analysis. Observed genotype frequencies for rs11614913 polymorphisms in controls were assessed for deviation from HWE using a goodness-of-fit Chi-square test with $\mathrm{df}=1$. $\mathrm{P}<0.05$ was considered representative of departure from HWE. Summary odds ratios (ORs) and $95 \%$ confidence intervals (CIs) were calculated for the allelic (C allele vs. T allele) and genotypic comparisons, following the co-dominant (CT vs. TT and CC vs. TT), dominant (CT+CC vs. TT) and recessive $(\mathrm{CC}$ vs. $\mathrm{TT}+\mathrm{CT})$ genetic models. For age comparison, the pooled weighted mean difference (WMD) was also performed. The significance of pooled ORs was determined by the Z-test and $\mathrm{P}<0.05$ was considered to indicate a statistically significant difference.

Statistical heterogeneity among the studies was assessed by the Chi-square-based Q-test. A Q-test P $>0.05$ indicated no significant heterogeneity among the studies (22) and the pooled OR was estimated by the fixed-effects model (Mantel-Haenszel method) (23). If the heterogeneity was significant, the randomeffects model (inverse variance method) was employed (24).

Publication bias was investigated with a funnel plot, which was used as the main graphical method. To supplement the funnel plot approach, the Begg and Mazumdar's adjusted rank correlation test (25) and the Egger's regression asymmetry test (26) were utilized.

Analyses were performed with Review Manager software (RevMan, version 5.0; The Cochrane Collaboration, Oxford, England) and Stata software, version 10.0 (StataCorp LP, College Station, TX, USA). All the P-values were two-sided. The statistical tests performed in the present analysis were considered to indicate a statistically significant difference whenever the corresponding null-hypothesis probability was $\mathrm{P}<0.05$.

\section{Results}

Characteristics of studies. Overall, six studies including 1,754 cases and 2,430 controls were available for this analysis. The study characteristics are provided in Table I. The sample size in these case-control studies varied considerably (338-1,161 individuals). There were four studies on Asian descendants and two on European descendants. Four genotyping methods were used, including Taqman, polymerase chain reaction-restriction fragment length polymorphism (PCR-RFLP), high-resolution melting analysis (HRMA) and polymerase chain reaction-ligation detection reaction (PCR-LDR). Furthermore, 83\% (5/6) of these studies included described genotyping quality control measures, such as a different genotyping assay to confirm the data and random repetition of a portion of samples. The genotype distributions among the controls of all studies were consistent with HWE.

In addition, the cases and controls of all the studies included in our analysis were matched by gender and age [no specific data were presented in the study by Vinci et al (20)]. The meta-analysis for age and gender detected no significant difference between the cases and the controls (for gender: $\mathrm{OR}=1.02$; 95\% CI: 0.89-1.17; heterogeneity test, $\mathrm{P}=0.46$, Fig. 1 ; for age: $\mathrm{WMD}=0.22$; $95 \% \mathrm{CI}$ : 0.69-1.14; heterogeneity test, $\mathrm{P}=0.08$ ), which suggested that age and gender were adequately matched in this meta-analysis.

Meta-analysis results. Six studies involving a total of 1,754 cases and 2,430 controls were assessed for the association between miR-196a2 rs11614913 polymorphism and CRC risk. There were no significant statistical heterogeneities in any of the comparison models; therefore, the fixed-effects model was used. Overall, the $\mathrm{C}$ allele were associated with a significantly increased risk when compared to the $\mathrm{T}$ allele (OR=1.14; 95\% CI: 1.04-1.24). Similarly, moderately elevated risks were also observed in overall analyses in the dominant ( $\mathrm{OR}=1.19 ; 95 \%$ CI: 1.03-1.39; Fig. 2) and the recessive model (OR=1.18; 95\% CI: 1.02-1.36). Moreover, the subgroup analysis in an Asian population also demonstrated similar results. Almost all the genetic models 
Table I. Characteristics of included studies that investigated the association between rs 11614913 polymorphisms of miR-196a2 and colorectal cancer risk.

\begin{tabular}{|c|c|c|c|c|c|c|c|c|c|c|c|c|c|c|}
\hline \multirow{2}{*}{$\begin{array}{l}\text { First } \\
\text { author }\end{array}$} & \multirow[b]{2}{*}{ Year } & \multirow[b]{2}{*}{ Country } & \multirow{2}{*}{$\begin{array}{l}\text { Ethnic } \\
\text { descent }\end{array}$} & \multirow[b]{2}{*}{ Genotyping } & \multirow{2}{*}{$\begin{array}{l}\text { Source of } \\
\text { control }\end{array}$} & \multirow{2}{*}{$\begin{array}{c}\text { Sample size } \\
\text { (case/control) }\end{array}$} & \multirow[b]{2}{*}{ P-value ${ }^{a}$} & \multicolumn{3}{|c|}{ Case } & \multicolumn{3}{|c|}{ Control } & \multirow[b]{2}{*}{ Refs } \\
\hline & & & & & & & & $\mathrm{CC}$ & $\mathrm{CT}$ & TT & $\mathrm{CC}$ & $\mathrm{CT}$ & TT & \\
\hline Chen et al & 2012 & China & Asian & PCR-LDR & HB & $126 / 407$ & 0.788 & 27 & 64 & 35 & 94 & 206 & 107 & (17) \\
\hline Hezova et al & 2012 & Czech & European & Taqman & HB & $197 / 212$ & 0.291 & 82 & 89 & 26 & 87 & 103 & 22 & (18) \\
\hline Min et al & 2012 & Korea & Asian & PCR-RFLP & PB & $446 / 502$ & 0.633 & 120 & 201 & 125 & 100 & 254 & 148 & (19) \\
\hline Vinci et al & 2013 & Italy & European & HRMA & HB & $160 / 178$ & 0.087 & 62 & 86 & 12 & 83 & 84 & 11 & (20) \\
\hline Zhan et al & 2011 & China & Asian & PCR-RFLP & HB & $252 / 543$ & 0.849 & 68 & 128 & 56 & 113 & 267 & 163 & (15) \\
\hline Zhu et al & 2012 & China & Asian & Taqman & HB & $573 / 588$ & 0.790 & 140 & 303 & 130 & 121 & 295 & 172 & (21) \\
\hline
\end{tabular}

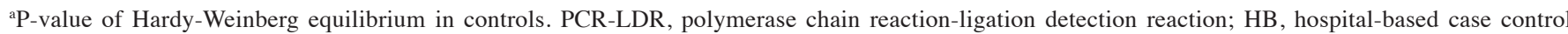
study; PCR-RFLP, polymerase chain reaction-restriction fragment length polymorphism; PB, population-based case control study; HRMA, high-resolution melting analysis.

\begin{tabular}{|c|c|c|c|c|c|c|c|c|c|}
\hline Study or Subgroup & $\begin{array}{l}\text { Case } \\
\text { Events }\end{array}$ & Total & $\begin{array}{l}\text { Contr } \\
\text { Events }\end{array}$ & Total & Weight & $\begin{array}{l}\text { Odds Ratio } \\
\mathrm{M}+\mathrm{H} \text {. Fixed. } 95 \% \mathrm{Cl}\end{array}$ & & $\begin{array}{c}\text { Odds Ratio } \\
\mathrm{M}+\mathrm{H} \text {. Fixed. } 95 \% \mathrm{Cl}\end{array}$ & \\
\hline Chen 2012 & 81 & 126 & 279 & 407 & $11.3 \%$ & $0.83[0.54,1.26]$ & & & \\
\hline Hezova 2012 & 105 & 197 & 93 & 202 & $10.3 \%$ & $1.34[0.90,1.98]$ & & & \\
\hline Min 2012 & 248 & 446 & 267 & 502 & $26.9 \%$ & $1.10[0.85,1.42]$ & & & \\
\hline Zhan 2011 & 174 & 252 & 383 & 543 & $18.1 \%$ & $0.93[0.67,1.29]$ & & & \\
\hline Zhu 2012 & 354 & 573 & 367 & 588 & $33.4 \%$ & $0.97[0.77,1.23]$ & & & \\
\hline Total $(95 \% \mathrm{Cl})$ & & 1594 & & 2242 & $100.0 \%$ & $1.02[0.89,1.17]$ & & & \\
\hline Total events & 962 & & 1389 & & & & & & \\
\hline \multicolumn{7}{|c|}{$\begin{array}{l}\text { Heterogeneity: } \mathrm{Chi}^{2}=3.59, \mathrm{df}=4(P=0.46) ;\left.\right|^{2}=0 \% \\
\text { Test for overall effect: } Z=0.31(P=0.76)\end{array}$} & 0.01 & 0.1 Case Control & 100 \\
\hline
\end{tabular}

Figure 1. Forest plot for gender between controls and cases of colorectal cancer.

\begin{tabular}{|c|c|c|c|c|c|c|c|c|c|c|}
\hline Study or Subgroup & $\begin{array}{l}\text { Case } \\
\text { Events }\end{array}$ & Total & $\begin{array}{l}\text { Contr } \\
\text { Events }\end{array}$ & $\begin{array}{l}\text { rol } \\
\text { Total }\end{array}$ & Weight & $\begin{array}{l}\text { Odds Ratio } \\
\text { MH. Fixed. } 95 \% \mathrm{Cl}\end{array}$ & & $\begin{array}{r}\text { Odds } \\
\text { M+H. Fixer }\end{array}$ & $\begin{array}{l}\text { s Ratio } \\
\text { ed. } 95 \% \mathrm{Cl}\end{array}$ & \\
\hline Chen 2012 & 91 & 126 & 300 & 407 & $12.5 \%$ & $0.93[0.59,1.45]$ & & & T & \\
\hline Hezova 2012 & 171 & 197 & 190 & 212 & $7.7 \%$ & $0.76[0.42,1.39]$ & & & t & \\
\hline Min 2012 & 321 & 446 & 354 & 502 & $29.6 \%$ & $1.07[0.81,1.42]$ & & & 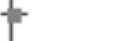 & \\
\hline Vinci 2013 & 148 & 160 & 167 & 178 & $3.8 \%$ & $0.81[0.35,1.90]$ & & & 一 & \\
\hline Zhan 2011 & 196 & 252 & 380 & 543 & $17.0 \%$ & $1.50[1.06,2.13]$ & & & 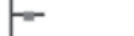 & \\
\hline Zhu 2012 & 443 & 573 & 416 & 588 & $29.5 \%$ & $1.41[1.08,1.83]$ & & & $=$ & \\
\hline Total $(95 \% \mathrm{CI})$ & & 1754 & & 2430 & $100.0 \%$ & $1.19[1.03,1.39]$ & & & 1 & \\
\hline Total events & 1370 & & 1807 & & & & & & & \\
\hline \multicolumn{7}{|c|}{$\begin{array}{l}\text { Heterogeneity: } \text { Chi }^{2}=7.85, \text { df }=5(P=0.16) ; 1^{2}=36 \% \\
\text { Test for overall effect: } Z=2.31(P=0.02)\end{array}$} & 0.01 & 0.1 Case $^{1}$ & ${ }^{1} 10$ & 100 \\
\hline
\end{tabular}

Figure 2. Forest plot of dominant model (CT+CC vs. TT) between miR-196a2 rs11614913 polymorphism and colorectal cancer.

indicated a significant association between rs11614913 polymorphism and CRC risk (C allele vs. T allele: $\mathrm{OR}=1.20$; 95\% CI: 1.08-1.32; CC vs. TT: OR=1.44; 95\% CI: 1.18-1.75; dominant model: $\mathrm{OR}=1.25$; $95 \% \mathrm{CI}$ : 1.06-1.46; recessive model: $\mathrm{OR}=1.30 ; 95 \% \mathrm{CI}: 1.10-1.53)$ except for the model of CT vs. TT. For CT vs. TT, there was no significant association in the overall analysis and the subgroup analysis in an Asian population. The results are shown in Table II.

The effect of miR-196a2 rs11614913 polymorphism was then evaluated based on the clinical characteristics of patients with CRC in three studies on the Han Chinese ethnic group $(15,17,21)$. The clinical CRC stage in two of those studies $(15,17)$ was evaluated on the basis of the TNM clas- sification system; however, in the third study (21) stage was classified into Dukes' A, B, C and D. It is well known that Dukes' $\mathrm{A}+\mathrm{B}$ is equivalent to clinical stage $\mathrm{I}+\mathrm{II}$ and Dukes' $\mathrm{C}+\mathrm{D}$ is equivalent to clinical stage III+IV [Ling-jun Zhu, the author of article (21), was contacted via e-mail and confirmed this classification]. Therefore, tumor stage was classified into two groups, III+IV vs. I+II. The tumor grade was also divided into two groups, intermediate/high vs. low. When we evaluated the associations between rs11614913 polymorphism and tumor stage or grade separately, no significant association was observed in any comparison model (Table III), which suggested that miR-196a2 rs11614913 polymorphism may not be associated with CRC stage or grade. 
Table II. Summary of ORs in the meta-analysis of SNP rs11614913 with the fixed-effects model.

\begin{tabular}{|c|c|c|c|c|c|}
\hline Genetic model & Population & No. of case/controls & OR $(95 \% \mathrm{CI})$ & P-value & $\mathrm{P}_{\mathrm{h}}$ \\
\hline \multirow[t]{2}{*}{$\mathrm{C}$ allele vs. $\mathrm{T}$ allele } & Overall & $3508 / 4860$ & $1.14(1.04-1.24)$ & 0.005 & 0.06 \\
\hline & Asian & $2794 / 4080$ & $1.20(1.08-1.32)$ & 0.0004 & 0.28 \\
\hline \multirow[t]{2}{*}{ CT vs. TT } & Overall & $1255 / 1832$ & $1.12(0.96-1.32)$ & 0.15 & 0.23 \\
\hline & Asian & $1042 / 1612$ & $1.17(0.99-1.38)$ & 0.07 & 0.19 \\
\hline \multirow[t]{2}{*}{ CC vs. TT } & Overall & $883 / 1221$ & $1.33(1.10-1.59)$ & 0.003 & 0.11 \\
\hline & Asian & $701 / 1018$ & $1.44(1.18-1.75)$ & 0.0003 & 0.28 \\
\hline Dominant model & Overall & $1754 / 2430$ & $1.19(1.03-1.39)$ & 0.02 & 0.16 \\
\hline$(\mathrm{CT}+\mathrm{CC}$ vs. TT $)$ & Asian & $1397 / 2040$ & $1.25(1.06-1.46)$ & 0.006 & 0.20 \\
\hline Recessive model & Overall & $1754 / 2430$ & $1.18(1.02-1.36)$ & 0.02 & 0.08 \\
\hline$(\mathrm{CC}$ vs. $\mathrm{TT}+\mathrm{CT})$ & Asian & $1397 / 2040$ & $1.30(1.10-1.53)$ & 0.002 & 0.38 \\
\hline
\end{tabular}

OR, odds ratio; SNP, single-nucleotide polymorphism; CI, confidence interval; $\mathrm{P}_{\mathrm{h}}$, P-value for heterogeneity test. The fixed-effects model was used when the P-value for heterogeneity test was $\geq 0.05$.

Table III. Meta-analyses of miR-196a2 rs11614913 polymorphism and clinical characteristics of colorectal cancer risk.

\begin{tabular}{|c|c|c|c|c|c|}
\hline Genetic model & Variables & No. of case/controls & OR $(95 \% \mathrm{CI})$ & P-value & $\mathrm{P}_{\mathrm{h}}$ \\
\hline \multirow[t]{2}{*}{$\mathrm{C}$ allele vs. $\mathrm{T}$ allele } & Tumor stage & $910 / 968$ & $1.10(0.92-1.32)$ & 0.29 & 0.23 \\
\hline & Tumor grade & $1172 / 610$ & $1.14(0.83-1.56)$ & 0.43 & 0.88 \\
\hline \multirow[t]{2}{*}{ CT vs. TT } & Tumor stage & $341 / 366$ & $1.28(0.93-1.76)$ & 0.13 & 0.16 \\
\hline & Tumor grade & $439 / 241$ & $0.90(0.52-1.58)$ & 0.72 & 0.59 \\
\hline \multirow[t]{2}{*}{ CC vs. TT } & Tumor stage & $208 / 238$ & $1.24(0.85-1.79)$ & 0.27 & 0.20 \\
\hline & Tumor grade & $281 / 141$ & $1.28(0.69-2.38)$ & 0.44 & 0.89 \\
\hline \multirow[t]{2}{*}{$\mathrm{CT}+\mathrm{CC}$ vs. TT } & Tumor stage & $455 / 484$ & $1.26(0.93-1.72)$ & 0.13 & 0.12 \\
\hline & Tumor grade & $586 / 305$ & $1.02(0.60-1.71)$ & 0.95 & 0.84 \\
\hline \multirow[t]{2}{*}{$\mathrm{CC}$ vs. TT+CT } & Tumor stage & $455 / 484$ & $1.04(0.77-1.40)$ & 0.80 & 0.72 \\
\hline & Tumor grade & $586 / 305$ & $1.38(0.82-2.31)$ & 0.23 & 0.52 \\
\hline
\end{tabular}

OR, odds ratio; $\mathrm{CI}$, confidence interval; $\mathrm{P}_{\mathrm{h}}, \mathrm{P}$-value for heterogeneity test.

Publication bias. Funnel plots, Egger's and Begg's tests were used to evaluate the publication bias of the literature on CRC. Symmetrical funnel plots were obtained in all the models. Egger's and Begg's tests further confirmed the absence of publication bias in this meta-analysis $(\mathrm{P}>0.05)$. Fig. 3 displays a funnel plot that assessed the miR-196a2 rs11614913 polymorphism and CRC risk in the dominant model.

\section{Discussion}

The association between miR-196a 2 rs11614913 polymorphisms and the risk of CRC has been previously investigated $(15,17-21)$. However, due to the limited sample size and the potential bias of case selection, these results were controversial and inconclusive. In this meta-analysis, we systematically summarized six eligible case-control studies on the association between SNP rs11614913 and the susceptibility to CRC.

The common genetic variant (rs11614913) was located in the $3 p$ mature miRNA region of hsa-miR-196a2. This $C>T$ polymorphism results in a change from G:C to G:T in the stem region of the miR-196a2 precursor. Several studies investigated the association between miR-196a 2 rs11614913 polymorphism and the miR-196a2 expression level in various types of cancer (13-15). Hoffman et al (13) demonstrated that mature miR-196a2 levels were increased 9.3-fold in cells transfected with pre-miR-196a2-C but only 4.4-fold with pre-miR-196a2-T. In the genotype-phenotype correlation analysis of cancer tissues, the $\mathrm{C}$ allele of rs11614913 increased the expression of mature miR-196a2 in lung cancer (14) and CRC tissues (15). These results indicated that rs11614913 polymorphism may affect the processing of the pre-miRNA to its mature form. It was previously reported that a high expression level of miR-196a may promote the migration and invasion of CRC cells (16) and recent studies reported the association of miR-196a2 rs11614913 polymorphism with CRC risk. For example, Zhan et al (15) first observed the association between SNP miR-196a2 rs11614913 and susceptibility to CRC. However, the results from other studies reported a lack of association of miR-196a2 rs11614913 and the risk for CRC $(17,18)$ or only associations between miR-196a2 SNP and the non-diabetic or rectal cancer groups (19). 


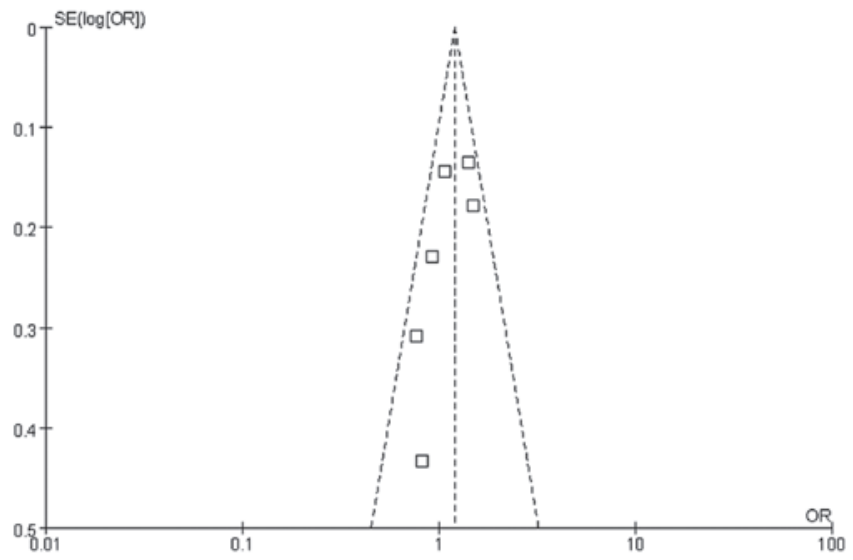

Figure 3. Begg's funnel plot for the publication bias test in the dominant model. SE, standard error; OR, odds ratio.

In this meta-analysis, six case-control studies were analyzed to provide a comprehensive assessment of the association between the miR-196a 2 rs11614913 polymorphism and CRC. Our results supported a genetic association between rs11614913 and susceptibility to CRC. It was observed that the hsa-miR-196a2 rs11614913 polymorphism was associated with an increased CRC risk in almost all the genetic models, except the model of CT vs. TT, indicating that the hsa-miR-196a2 rs11614913 polymorphism may be important in the development of CRC. Since the incidence of gene polymorphisms may vary between different ethnic groups and this variation may interfere with the detection of minor effects of SNPs on CRC risk, a subgroup analysis in an Asian population was performed to further investigate the potential association between rs11614913 and the risk of CRC. The subgroup analysis also demonstrated a significant association of miR-196a2 rs11614913 polymorphism with susceptibility to CRC. Moreover, since the clinical characteristics of patients were well-established risk factors for the development of CRC, we also evaluated the effect of rs11614913 polymorphism on CRC by a stratified analysis of tumor stage and grade. However, there was no association identified between rs11614913 polymorphism and the risk for clinical characteristics of patients in any of the genetic models, suggesting that tumor stage and grade may not be the main factors affecting the stability of these comparisons. It also indicated that rs11614913 polymorphism may be involved in the occurrence of CRC, but not in its progression. The sensitivity analysis did not detect a significant effect of any single study on pooled ORs, indicating that the stability of this meta-analysis was acceptable.

Ten previous meta-analyses reviewed the potential role of polymorphism rs11614913 in the development of cancer, seven of which reported a statistically significant association between this polymorphism and susceptibility to cancer without pre-specified tissue origin (27-33) and three focused on a pre-specified cancer type, such as breast cancer (34), hepatocellular carcinoma (35) or digestive system cancer (36). However, clinical heterogeneity due to inherent differences between cancers of distinct tissue origins may limit the reliability of the conclusions of those meta-analyses (35). Therefore, a meta-analysis focusing on one specific type of cancer may increase the stability of the conclusions. Thus far, only two meta-analyses investigated the association between rs11614913 and CRC by means of subgroup analysis $(32,36)$. Those results were consistent with ours in the allele frequency comparison ( $\mathrm{C}$ allele vs. $\mathrm{T}$ allele), co-dominant model (CC vs. TT) and recessive model (CC vs. TT+CT). However, the meta-analysis of Wang et al (32) analyzed three case-control studies and reported a moderately elevated risk in the dominant model, which was consistent with our findings but inconsistent with those of Guo et al (36). Similarly, the two subgroup results $(32,36)$ indicated that rs11614913 C/T heterozygosity is significantly associated with the risk of CRC, which was inconsistent with our results. These inconsistencies may be partly due to the fact that none of the meta-analyses included all the available studies on the association between rs11614913 and susceptibility to CRC.

To the best of our knowledge, this is the first meta-analysis evaluating the potential association between rs11614913 in miR-196a2 and susceptibility to CRC. It has been well established that environmental factors, dietary habits and lifestyle habits, such as drinking and smoking, are associated with increased risk of CRC $(2,3,37)$. In addition, a positive family history may play a key role in CRC risk $(38,39)$. However, lack of available data prevented an adjustment for subgroup factors, such as family history of CRC, alcohol consumption and smoking status. However, our meta-analysis held some key benefits. First, no heterogeneity was detected in any comparison and the sensitivity analysis demonstrated that none of the studies exerted a significant effect on the evaluation of potential association, which indicated that the results were more reliable. Second, the evaluation of rs11614913 polymorphism and the risk of patient clinical characteristics were evaluated in our study, but not in those of Wang et al (32) and Guo et al (36). The stratified analysis in our study may have enhanced the stability of the results and the reliability of the conclusions. Thirdly, the cases and controls of all the studies included in our analysis were adequately matched by gender and age, which ensured the stability of our results and conclusions.

In summary, miR-196a2 rs11614913 polymorphisms may be associated with the risk of CRC. Well-designed studies including larger sample sizes and more ethnic groups are required to further elucidate this association. Other factors, such as gender, age, smoking status, tumor stage and grade, lymph node status and tumor invasiveness should also be considered in future studies.

\section{Acknowledgements}

We would like to thank Guicheng Zhang (School of Paediatrics and Child Health, University of Western Australia, Australia) for his helpful comments. This study was supported by the Natural Science Foundation of China (grant no. 81172333).

\section{References}

1. Schnekenburger M and Diederich M: Epigenetics offer new horizons for colorectal cancer prevention. Curr Colorectal Cancer Rep 8: 66-81, 2012.

2. Glade MJ: Food, nutrition, and the prevention of cancer: a global perspective. American Institute for Cancer Research/World Cancer Research Fund, American Institute for Cancer Research, 1997. Nutrition 15: 523-526, 1999. 
3. Potter JD: Colorectal cancer: molecules and populations. J Natl Cancer Inst 91: 916-932, 1999.

4. Jasperson KW, Tuohy TM, Neklason DW and Burt RW: Hereditary and familial colon cancer. Gastroenterology 138 2044-2058, 2010.

5. Burt RW: Colon cancer screening. Gastroenterology 119: $837-853,2000$

6. Lichtenstein P, Holm NV, Verkasalo PK, Iliadou A, Kaprio J Koskenvuo M, Pukkala E, Skytthe A and Hemminki K: Environmental and heritable factors in the causation of cancer - analyses of cohorts of twins from Sweden, Denmark, and Finland. N Engl J Med 343: 78-85, 2000.

7. Bartel DP: MicroRNAs: genomics, biogenesis, mechanism, and function. Cell 116: 281-297, 2004.

8. Esquela-Kerscher A and Slack FJ: Oncomirs - microRNAs with a role in cancer. Nat Rev Cancer 6: 259-269, 2006.

9. Vasudevan S, Tong Y and Steitz JA: Switching from repression to activation: microRNAs can up-regulate translation. Science 318 : 1931-1934, 2007

10. Loktionov A: Common gene polymorphisms, cancer progression and prognosis. Cancer Lett 208: 1-33, 2004.

11. Duan R, Pak C and Jin P: Single nucleotide polymorphism associated with mature miR-125a alters the processing of pri-miRNA. Hum Mol Genet 16: 1124-1131, 2007.

12. Zeng Y and Cullen BR: Sequence requirements for micro RNA processing and function in human cells. RNA 9: 112-123, 2003.

13. Hoffman AE, Zheng T, Yi C, Leaderer D, Weidhaas J, Slack F, Zhang Y, Paranjape T and Zhu Y: microRNA miR-196a-2 and breast cancer: a genetic and epigenetic association study and functional analysis. Cancer Res 69: 5970-5977, 2009.

14. Hu Z, Chen J, Tian T, Zhou X, Gu H, Xu L, Zeng Y, Miao R, Jin G, Ma H, et al: Genetic variants of miRNA sequences and non-small cell lung cancer survival. J Clin Invest 118: 2600-2608, 2008.

15. Zhan JF, Chen LH, Chen ZX, Yuan YW, Xie GZ, Sun AM and Liu Y: A functional variant in microRNA-196a2 is associated with susceptibility of colorectal cancer in a Chinese population. Arch Med Res 42: 144-148, 2011.

16. Schimanski CC: High miR-196a levels promote the oncogenic phenotype of colorectal cancer cells. World J Gastroenterol 15: 2089-2096, 2009.

17. Chen H, Sun LY, Chen LL, Zheng HQ and Zhang QF: A variant in microRNA-196a2 is not associated with susceptibility to and progression of colorectal cancer in Chinese. Intern Med J 42 : e115-e119, 2012

18. Hezova R, Kovarikova A, Bienertova-Vasku J, Sachlova M, Redova M, Vasku A, Svoboda M, Radova L, Kiss I, Vyzula R and Slaby O: Evaluation of SNPs in miR-196-a2, miR-27a and miR-146a as risk factors of colorectal cancer. World J Gastroenterol 18: 2827-2831, 2012

19. Min KT, Kim JW, Jeon YJ, Jang MJ, Chong SY, Oh D and Kim NK: Association of the miR-146aC $>$ G, 149C $>$ T, 196a2C $>$ T, and $499 \mathrm{~A}>\mathrm{G}$ polymorphisms with colorectal cancer in the Korean population. Mol Carcinog 51 (Suppl 1): E65-E73, 2012.

20. Vinci S, Gelmini S, Mancini I, Malentacchi F, Pazzagli M, Beltrami C, Pinzani P and Orlando C: Genetic and epigenetic factors in regulation of microRNA in colorectal cancers. Methods 59: 138-146, 2013

21. Zhu L, Chu H, Gu D, Ma L, Shi D, Zhong D, Tong N, Zhang Z and Wang M: A functional polymorphism in miRNA-196a 2 is associated with colorectal cancer risk in a Chinese population. DNA Cell Biol 31: 350-354, 2012.
22. Lau J, Ioannidis JP and Schmid CH: Quantitative synthesis in systematic reviews. Ann Intern Med 127: 820-826, 1997.

23. Mantel $\mathrm{N}$ and Haenszel W: Statistical aspects of the analysis of data from retrospective studies of disease. J Natl Cancer Inst 22: 719-748, 1959.

24. DerSimonian R and Laird N: Meta-analysis in clinical trials. Control Clin Trials 7: 177-188, 1986.

25. Begg CB and Mazumdar M: Operating characteristics of a rank correlation test for publication bias. Biometrics 50: 1088-1101, 1994.

26. Egger M, Davey Smith G, Schneider M and Minder C: Bias in meta-analysis detected by a simple, graphical test. BMJ 315: 629-634, 1997.

27. Wang P, Xie S, Cui A, Zhang Y and Jiang B: miR-196a2 polymorphisms and susceptibility to cancer: A meta-analysis involving 24,697 subjects. Exp Ther Med 3: 324-330, 2012.

28. Wang F, Ma YL, Zhang P, Yang JJ, Chen HQ, Liu ZH, Peng JY, Zhou YK and Qin HL: A genetic variant in microRNA-196a2 is associated with increased cancer risk: a meta-analysis. Mol Biol Rep 39: 269-275, 2012.

29. Chu H, Wang M, Shi D, Ma L, Zhang Z, Tong N, Huo X, Wang W, Luo D, Gao Y and Zhang Z: Hsa-miR-196a2 Rs11614913 polymorphism contributes to cancer susceptibility: evidence from 15 case-control studies. PLoS One 6: e18108, 2011.

30. Xu W, Xu J, Liu S, Chen B, Wang X, Li Y, Qian Y, Zhao W and $\mathrm{Wu} \mathrm{J}$ : Effects of common polymorphisms rs11614913 in miR-196a2 and rs2910164 in miR-146a on cancer susceptibility: a meta-analysis. PLoS One 6: e20471, 2011.

31. Qiu LX, Wang Y, Xia ZG, Xi B, Mao C, Wang JL, Wang BY, Lv FF, Wu XH and Hu LQ: miR-196a 2 C allele is a low-penetrant risk factor for cancer development. Cytokine 56: 589-592, 2011.

32. Wang J, Wang Q, Liu H, Shao N, Tan B, Zhang G, Wang K, Jia Y, Ma W, Wang N and Cheng Y: The association of miR-146a rs2910164 and miR-196a2 rs11614913 polymorphisms with cancer risk: a meta-analysis of 32 studies. Mutagenesis 27: 779-788, 2012

33. Tian T, Xu Y, Dai J, Wu J, Shen H and Hu Z: Functional polymorphisms in two pre-microRNAs and cancer risk: a meta-analysis. Int J Mol Epidemiol Genet 1: 358-366, 2010.

34. Gao LB, Bai P, Pan XM, Jia J, Li LJ, Liang WB, Tang M, Zhang LS, Wei YG and Zhang L: The association between two polymorphisms in pre-miRNAs and breast cancer risk: a meta-analysis. Breast Cancer Res Treat 125: 571-574, 2011.

35. Wang Z, Cao Y, Jiang C, Yang G, Wu J and Ding Y: Lack of association of two common polymorphisms rs2910164 and rs11614913 with susceptibility to hepatocellular carcinoma: a meta-analysis. PLoS One 7: e40039, 2012.

36. Guo J, Jin $M$, Zhang $M$ and Chen $K$ : A genetic variant in miR-196a2 increased digestive system cancer risks: a meta-analysis of 15 case-control studies. PLoS One 7: e30585, 2012.

37. Zambirinis CP, Theodoropoulos G and Gazouli M: Undefined familial colorectal cancer. World J Gastrointest Oncol 1: 12-20, 2009.

38. Burt R: Inheritance of colorectal cancer. Drug Discov Today Dis Mech 4: 293-300, 2007.

39. Patel SG and Ahnen DJ: Familial colon cancer syndromes: an update of a rapidly evolving field. Curr Gastroenterol Rep 14: 428-438, 2012 